Comparação da Eficiência de Diferentes Técnicas (Esgotamento e Foulardagem) de Fixação de Permetrina em Tecidos Têxteis

\author{
Martins, T. G.; Chiapetta, S. C.; Carvalho, L. J.; Cassella, R. J.
}

Rev. Virtual Quim., 2015, 7 (4), 1119-1129. Data de publicação na Web: 21 de fevereiro de 2015

\author{
http://www.uff.br/rvq
}

\title{
Comparison of the Eficiency of Different Techniques (Exhaustion and Padding) for the Fixation of Permethrin in Fabrics
}

\begin{abstract}
Due to lack of knowledge of chemoprophylaxis for most diseases transmitted by insects, the use of repellent is considered one of the most important means in terms of combating insect bites and the diseases transmitted that way. According to this trend, the use of repellents applied directly to the skin, and also impregnated into the fabrics used for making clothes have been frequently used and considered of great importance. Permethrin is a species commonly used for this purpose and such possibility exists due to two important facts: it has low toxicity by dermal adsorption and it presents high efficiency in repelling action. Several manufacturing methods are used to fix chemicals into the tissue. In this work, two different methods of fixation of permethrin into tissues were evaluated for their efficiency: exhaustion and padding. In the process of exhaustion, the fabric remains under stirring in bath, in contact with the solution of permethrin. In process of padding, the fabric passes through rollers so that the solution is forced to enter into it. The efficiency of fixation was assessed by quantificating impregnated permethrin and the results obtained indicated that exhaustion procedure was more efficient, when using the same initial concentration of permethrin at both processes, a greater concentration into the fabrics was achieved by exhaustion.
\end{abstract}

Keywords: Permethrin; Depletion; Padding.

\section{Resumo}

Devido à falta de conhecimento em quimioprofilaxia para a maioria das doenças transmitidas por picada de inseto, considera-se a utilização de repelentes como um dos itens de maior importância no combate a doenças assim transmitidas. Seguindo essa tendência, a utilização, não apenas dos repelentes aplicados diretamente na pele, mas também dos impregnados nos tecidos utilizados para fabricação de roupas tem sido frequente e de grande importância. A permetrina é uma das espécies comumente utilizada para esse fim e tal possibilidade existe devido ao fato da mesma possuir baixa toxidade por adsorção cutânea aliada a sua alta eficiência na ação repelente. Diversos são os métodos industriais utilizados com a finalidade de fixar uma substância química no tecido. Nesse trabalho, dois métodos diferentes de fixação de permetrina em tecido foram avaliados quanto a sua eficiência: o esgotamento e a foulardagem. No processo de esgotamento, o tecido permanece sob agitação em contato com o banho da solução de permetrina, e na foulardagem, o tecido passa por roletes compressores para que essa solução seja forçada a penetrar no mesmo. A eficiência da fixação foi avaliada pela quantificação da permetrina nos tecidos impregnados e os resultados obtidos indicaram que o procedimento por esgotamento mostrou-se mais eficiente, uma vez que foi encontrada uma maior concentração de permetrina no tecido quando fixado por essa técnica.

Palavras-chave: Permetrina; Esgotamento; Foulardagem.

* Universidade Federal Fluminense, Instituto de Química, Departamento de Química Analítica, Campus do Valonguinho, CEP 24020-141, Niterói-RJ, Brasil.

Mtayene.quimica@yahoo.com.br

DOI: $\underline{10.5935 / 1984-6835.20150062}$ 


\section{Comparação da Eficiência de Diferentes Técnicas (Esgotamento} e Foulardagem) de Fixação de Permetrina em Tecidos Têxteis

\section{Tayene G. Martins, ${ }^{\mathrm{a}, \mathrm{b}, *}$ Simone C. Chiapetta, ${ }^{\mathrm{b}}$ Lucas J. Carvalho, ${ }^{\mathrm{c}}$ Ricardo J. Cassella ${ }^{\mathrm{a}}$}

a Universidade Federal Fluminense, Instituto de Química, Departamento de Química Analítica, Campus do Valonguinho, CEP 24020-141, Niterói-RJ, Brasil.

${ }^{\mathrm{b}}$ Instituto Nacional de Tecnologia, Divisão de Química Analítica, Laboratório de Tabaco e Derivados, Rio de Janeiro-RJ, Brasil.

'Instituto Nacional de Metrologia, Qualidade e Tecnologia, Diretoria de Metrologia Científica e Industrial, Divisão de Química, Laboratório de Análise Orgânica, Duque de Caxias-RJ, Brasil.

* tayene.quimica@yahoo.com.br

Recebido em 29 de junho de 2014. Aceito para publicação em 12 de fevereiro de 2015

\section{Introdução}

1.1. As técnicas de fixação

\section{Materiais e Métodos}

2.1. Instrumentação

2.2. Reagentes e soluções

2.3. Solução padrão de permetrina

2.4. Tecidos

2.5. Gramatura dos tecidos

2.6. Metodologias

2.7. Condições Cromatográficas

\section{Resultados e Discussão}

4. Conclusão

\section{Introdução}

Mosquitos são transmissores de doenças para aproximadamente 700 milhões de pessoas por ano. ${ }^{1}$ Mais de 3000 espécies de mosquitos são conhecidas, ainda que se desconheçam os meios para se combater aquelas espécies prejudiciais ao homem. ${ }^{2}$
Algumas doenças passaram a ser transmitidas em áreas urbanas, graças à introdução de seus vetores nessas áreas. ${ }^{3}$

No Brasil, algumas doenças vetoriais possuem maior ocorrência, tais como a dengue, a malária, a leishmaniose e a doença de Chagas. Em razão da importância epidemiológica e do grau de participação do vetor no processo de transmissão, torna-se 
necessária a correta identificação dos diferentes tipos de vetores envolvidos na disseminação destas doenças. ${ }^{3}$

As estratégias de controle das doenças transmissíveis por vetores são bastante complexas, principalmente devido à existência de reservatórios domésticos e silvestres e aos aspectos ambientais. $O$ amplo uso dos inseticidas sintéticos para o controle de pragas domésticas e na agricultura, desde a descoberta do Dicloro-Difenil-Tricloroetano (DDT), levou a uma maior preocupação em relação à toxicidade e impacto ambiental destas substâncias. ${ }^{4}$ Os principais problemas associados ao uso dos inseticidas são o emprego de maiores concentrações, na tentativa de recuperar a eficácia, o aumento da frequência de aplicação e a substituição do produto. Além de essas ações serem ambientalmente negativas, não são eficazes no controle da expansão territorial dos insetos vetores..$^{5} \mathrm{O}$ uso indiscriminado dos inseticidas pode ser uma das causas da existência de vetores resistentes a determinados produtos químicos, bem como fatores genéticos e outros.

Em geral, a eficiência dos inseticidas é reduzida gradualmente com o tempo, uma vez que os insetos acabam por desenvolver resistência aos mesmos. Fatores operacionais tais como o tipo de inseticida, a sua concentração, o método e a frequência de aplicação, também podem desempenhar importante papel no desenvolvimento desta resistência. ${ }^{6}$ Assim, a necessidade de desenvolvimento de novas moléculas e formulações mais adequadas tem impulsionado as pesquisas nesta área e tem feito crescer o interesse por inseticidas alternativos. $^{7}$

A combinação da aplicação tópica do $N, N$ dietil-m-toluamida (DEET) e a impregnação de roupas por permetrina compreende a forma mais recomendada de repelência de mosquitos em adultos. ${ }^{8,9}$ Apesar de ser uma potente neurotoxina para insetos, a toxicidade da permetrina em mamíferos é considerada baixa, promovendo o seu uso generalizado em controle de pragas em medicina veterinária e humana. ${ }^{10,11}$

A permetrina é um agroquímico pertencente à classe dos inseticidas sintéticos piretroides. Sua eficácia e segurança na profilaxia de infestações cutâneas e controle de ervas daninhas são conhecidos por dermatologistas e agricultores há décadas. ${ }^{12,13}$ Os piretroides podem ser naturais ou sintéticos. Os piretroides naturais podem ser obtidos a partir da piretrina presente nas flores do crisântemo, enquanto os sintéticos são obtidos a partir de modificações estruturais da piretrina. ${ }^{14}$

A permetrina, desenvolvida por Elliott et al. em $1973,{ }^{15}$ vem sendo utilizada desde então para controlar a pediculose. Entretanto, a sua utilização foi intensificada com a introdução de produtos "over the counter" (OTC), mais comumente conhecidos como produtos de prateleira, na década de $1980 .{ }^{16}$ A permetrina representa um quarto dos inseticidas utilizados na agricultura em todo o mundo. Porém, os derivados de piretrinas naturais têm uso limitado na agricultura devido à baixa fotoestabilidade, enquanto os piretroides sintéticos são fotoestáveis e altamente efetivos contra um largo espectro de insetos. Ainda, apresentam baixa toxicidade para os mamíferos. ${ }^{17}$

As estruturas das substâncias permetrina e piretrina estão apresentadas na Figura 1. A permetrina é frequentemente encontrada como uma mistura isomérica. Em geral, o isômero $Z$ é encontrado em menor proporção e possui maior toxicidade que seu isômero $E$, que é metabolizado mais rapidamente no organismo, sendo assim menos tóxico para os mamíferos. ${ }^{13}$ 


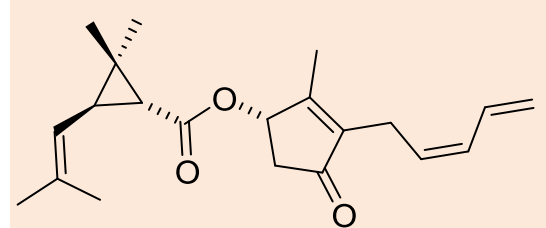

Piretrina

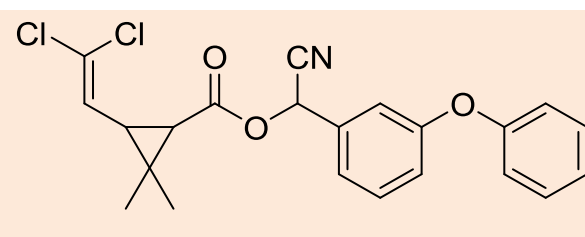

Permetrina

Figura 1. Estruturas moleculares da permetrina e piretrina

\subsection{As técnicas de fixação}

A impregnação de roupas pode ser efetuada através de várias técnicas. Uma destas, chamada de esgotamento, consiste na imersão do tecido em uma solução contendo a espécie a ser impregnada (nesse caso, a permetrina) por um tempo definido. Nesse estudo, parâmetros como o tempo de contato, concentração de permetrina na solução e temperatura do banho foram otimizados.

Esse contato forçaria a adsorção da permetrina sobre a superfície do tecido. No entanto, tanto pelo fato da transferência de massa quanto a adsorção serem pouco eficientes, ocorre uma distribuição desigual da permetrina no tecido e uma perda muito grande nas primeiras lavagens. ${ }^{18}$ Para melhorar a fixação e evitar essas perdas, os banhos de permetrina utilizados na indústria contém um polímero fixador, empregado para que ocorra a formação de um filme polimérico capaz de fixar a permetrina no tecido. ${ }^{19,20}$

Outra técnica convencional de impregnação utilizada é a foulardagem. Ela compreende um processo onde o banho é praticamente "forçado" a penetrar no interior do material têxtil a partir da força exercida por dois cilindros, como visto na Figura 2. Este processo costuma melhorar a transferência de massa, o que tornaria o processo de adsorção mais eficiente. ${ }^{21}$ Por sua vez, o baixo tempo de contato entre a solução e o tecido, na foulardagem, prejudicaria a adsorção de grandes quantidades de inseticida sobre o tecido.

No processo de foulardagem também se faz uso de polímeros fixadores para melhorar a adsorção da permetrina. Nos dois processos, a eficiência da adsorção pode ser influenciada pela temperatura do banho e pela temperatura de secagem.

Outras técnicas de impregnação (PADsteam, PAD-batch, PAD-dry, PAD-dry:steam, etc.) também podem ser empregadas para este fim. Entretanto, devido ao mais elevado grau de complexidade das operações envolvidas, não são utilizadas com frequência em indústrias de pequeno porte.

Uma forma de avaliar a eficiência do processo é determinando-se a menor concentração de permetrina no banho suficiente para se alcançar a adsorção de uma quantidade adequada do inseticida no tecido. Processos de fixação muito eficientes necessitam de menores concentrações de permetrina no banho para atingir o mesmo resultado, o que gera um custo operacional menor.

O objetivo deste trabalho foi realizar um estudo para avaliação da eficiência de dois dos processos mais comuns empregados na indústria para impregnação de permetrina em tecidos: a foulardagem e o esgotamento. Esse estudo foi realizado determinando-se a concentração de permetrina impregnada em tecidos submetidos a cada um dos dois processos. 


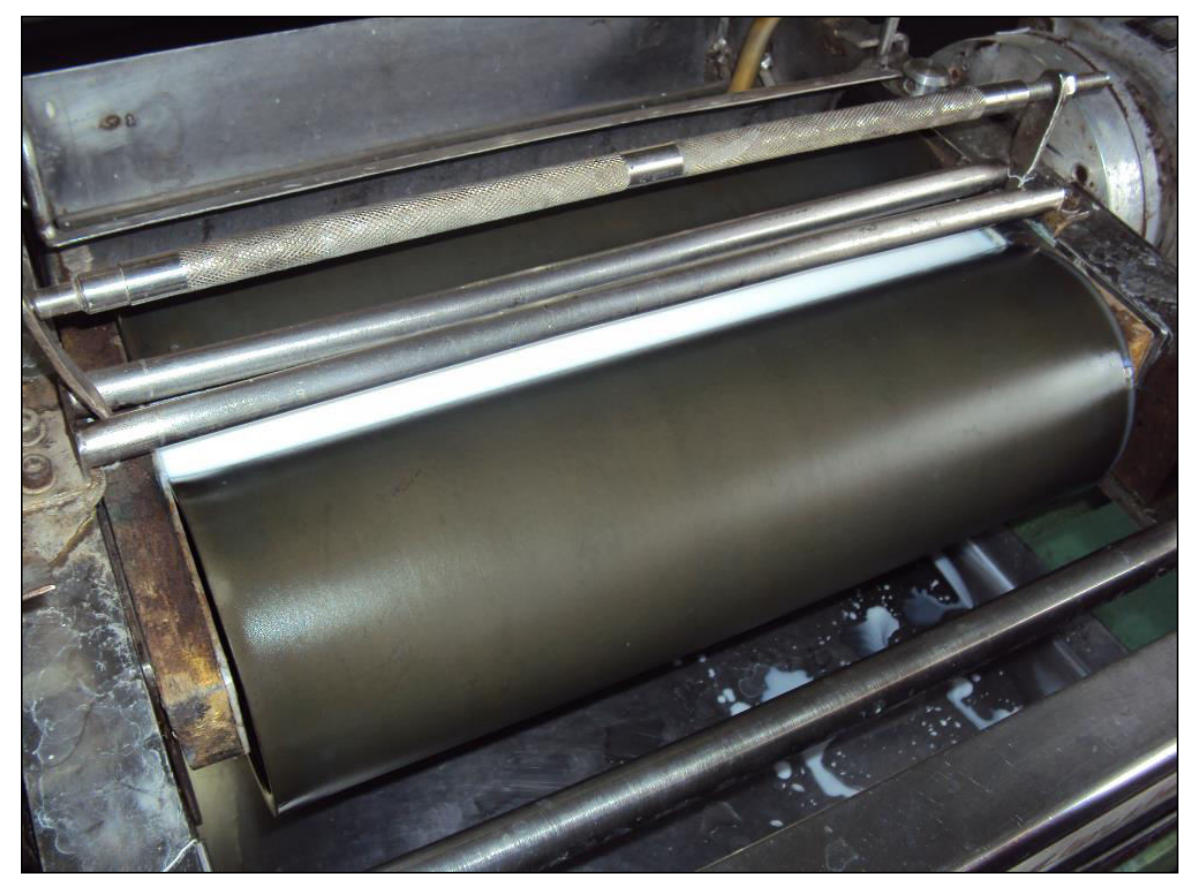

Figura 2. Máquina utilizada para o processo de foulardagem

\section{Materiais e Métodos}

\subsection{Instrumentação}

Amostras e padrões foram analisados utilizando-se um cromatógrafo a líquido de ultra eficiência (CLUE) equipado com detector de arranjo de diodos (Waters). Para a extração da permetrina dos tecidos avaliados foi utilizado um banho de ultrassom (Ultracleaner 1600, da marca Unique). A pesagem das amostras foi realizada em balança analítica (marca Shimadzu, modelo AY220, com precisão de $0,1 \mathrm{mg}$ ) e o foulard utilizado na impregnação dos tecidos foi um modelo MWF033 da Delta Equipamentos.

\subsection{Reagentes e soluções}

A fase móvel utilizada nas separações cromatográficas foi preparada pela mistura de metanol e acetonitrila. Ambos os solventes (grau HPLC) foram adquiridos da Merck.
Para o preparo da solução de permetrina para aplicação nos tecidos utilizou-se uma solução industrial de permetrina (50\%, valor nominal) e uma solução industrial de fixador polimérico. A composição do fixador era desconhecida, uma vez que é segredo industrial. As soluções de fixador e inseticida foram preparadas em água. O padrão de permetrina empregado na quantificação foi adquirido na Sigma-Aldrich, e tratava-se de uma mistura isomérica (25:75\%, $Z$ e $E$ respectivamente). Todas as soluções padrão empregadas foram preparadas em metanol.

\subsection{Solução padrão de permetrina}

Para a construção da curva analítica de permetrina preparou-se uma solução estoque de $40 \mathrm{mg} \cdot \mathrm{L}^{-1}$ de permetrina em metanol. A partir desta solução, procederamse diluições, também com metanol, de modo a obter soluções com as concentrações de 2,5; 5,0; 10,0; 20,0 mg. $\mathrm{L}^{-1}$ de permetrina. 


\subsection{Tecidos}

Os tecidos utilizados no estudo da aplicação dos diferentes procedimentos de impregnação possuíam composição $100 \%$ algodão, cor branca e tamanho 210 mm x 297 $\mathrm{mm}$.

\subsection{Gramatura dos tecidos}

Como a gramatura do tecido é um parâmetro que influencia em sua capacidade de sofrer impregnação, os tecidos estudados foram amostrados de forma a possuir a mesma gramatura. Os tecidos foram cortados para possuírem dimensões de $5 \times 5 \mathrm{~cm}$, sua massa pesada, e a gramatura foi calculada multiplicando por 400 o valor dessa massa, obtendo assim a gramatura em $\mathrm{mg} \cdot \mathrm{m}^{-2}$.

\subsection{Metodologias}

- Impregnação dos tecidos por
esgotamento

A impregnação pelo processo de esgotamento foi aplicada a um tecido com composição $100 \%$ algodão, no tamanho $20 \mathrm{x}$ $30 \mathrm{~cm}$. A solução do banho foi preparada com concentração de $18 \mathrm{~g} \cdot \mathrm{L}^{-1}$ de permetrina e $10 \mathrm{~g}^{-1} \mathrm{~L}^{-1}$ de fixador e a temperatura mantida a $60{ }^{\circ} \mathrm{C}$ durante todo o processo. $\mathrm{O}$ tecido foi mantido sob agitação e em contato com o banho durante $20 \mathrm{~min}$, após os quais foi retirado do banho e levado à estufa para secagem por $10 \mathrm{~min}$ a $130{ }^{\circ} \mathrm{C}$.
- Impregnação do tecido por foulardagem

A impregnação pelo processo de foulardagem foi aplicada ao mesmo tecido utilizado para impregnação por esgotamento, com composição $100 \%$ de algodão e com tamanho $20 \times 30 \mathrm{~cm}$. A solução empregada no processo de impregnação apresentava concentração de permetrina de $18 \mathrm{~g} \cdot \mathrm{L}^{-1} \mathrm{e}$ concentração de fixador de $10 \mathrm{~g} \cdot \mathrm{L}^{-1}$. A solução foi aplicada à junção dos dois roletes compressores, por onde passava o tecido. Após a passagem pelos roletes, os tecidos foram levados à estufa para secagem por 10 $\min a 130^{\circ} \mathrm{C}$.

- Metodologia para extração da
permetrina do tecido

Cerca de $0,5 \mathrm{~g}$ de tecido foram pesados diretamente em tubo de polietileno com 15 $\mathrm{mL}$ de capacidade e, em seguida, foram adicionados $10 \mathrm{~mL}$ de metanol. O tubo foi levado ao banho ultrassom por 15 min para sonicação visando o aumento da eficiência de extração. A solução obtida foi filtrada em membrana de PTFE com 0,22 $\mu \mathrm{m}$ de diâmetro de poro. A solução filtrada foi diluída 15 vezes e injetada no sistema CLUE DAD.

\subsection{Condições Cromatográficas}

Os parâmetros cromatográficos utilizados para determinação de permetrina nos extratos e soluções padrão foram adaptados de GARCIA et al., ${ }^{22}$ em que foi realizada a quantificação de permetrina em formulações farmacêuticas por CLUE-DAD. Tais parâmetros são apresentados na Tabela 1. 
Tabela 1. Condições cromatográficas para determinação de permetrina

\begin{tabular}{cc}
\hline \multicolumn{1}{c}{ Condições Cromatográficas } \\
\hline Fase Móvel & MeOH:água $(78: 22 \%, v / \mathrm{v})$ \\
Temperatura da coluna & $35^{\circ} \mathrm{C}$ \\
Vazão de fase móvel & $0,5 \mathrm{~mL} \cdot \mathrm{min}^{-1}$ \\
Comprimento de onda & $210 \mathrm{~nm}$ \\
Volume de Injeção & $2,0 \mu \mathrm{L}$ \\
Coluna cromatográfica & $\mathrm{C}_{18} \mathrm{HSS}$ (Acquity UPLC HSS, $50 \times 2,1 \mathrm{~mm}, 1,8 \mu \mathrm{m}$ ) \\
Tempo de análise & $5,0 \mathrm{~min}$ \\
\hline
\end{tabular}

\section{Resultados e Discussão}

A primeira etapa do trabalho foi avaliar as características analíticas do método cromatográfico selecionado para a determinação de permetrina nos extratos metanólicos. Nas condições otimizadas, o método cromatográfico permitiu a construção de curvas de calibração no intervalo de concentrações de 4,0 a 45,0 $\mathrm{mg} \cdot \mathrm{L}^{-1}$. Todas as curvas construídas ao longo do trabalho apresentaram linearidade satisfatória $\left(r^{2}>0,999\right)$. Os limites de detecção (LD) e de quantificação (LQ), em solução, foram obtidos a partir de dez medições do branco e foram iguais a $0,22 \mathrm{e}$ $0,74 \mathrm{mg} \cdot \mathrm{L}^{-1}$, respectivamente. Levando-se em conta o procedimento de extração, o método apresentou um LD de $0,88 \mathrm{mg} \cdot \mathrm{m}^{-2}$ e um LQ de $2,9 \mathrm{mg} \cdot \mathrm{m}^{-2}$. As curvas de calibração foram construídas diariamente. A Figura 3 apresenta uma curva de calibração típica, construída com soluções padrão de permetrina em metanol.

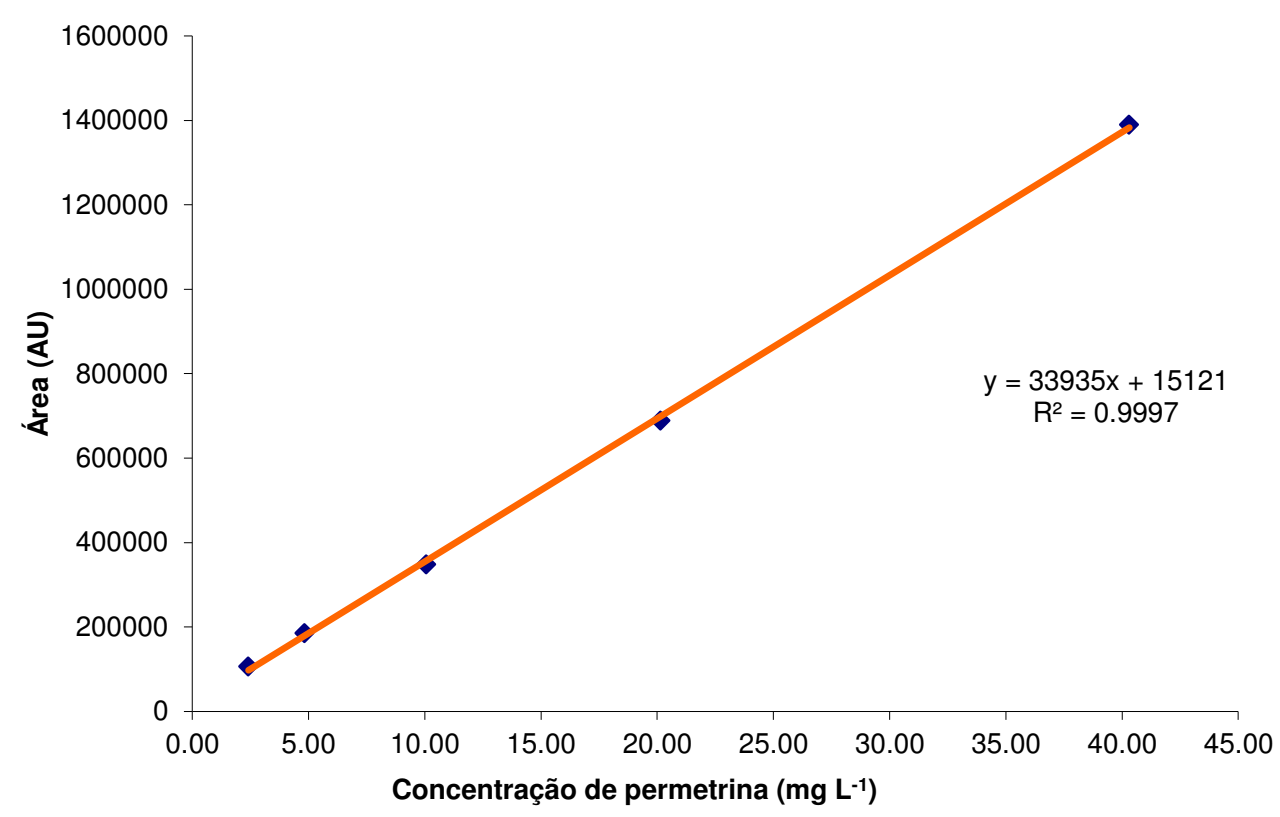

Figura 3. Curva de calibração utilizada na quantificação de permetrina na amostra II

O procedimento de extração aplicado permitiu a determinação da permetrina por CLUE-DAD em todos os extratos de tecidos testados. As Figuras 4 e 5 apresentam respectivamente, exemplos de perfis cromatográficos de uma solução padrão de 
permetrina em metanol com concentração de 20 mg. L $^{-1}$ e um exemplo de um cromatograma de um extrato obtido de um tecido impregnado com permetrina. A detecção foi realizada em $210 \mathrm{~nm}$. Por essas figuras pode-se observar que os cromatogramas obtidos na solução padrão e no extrato são idênticos.

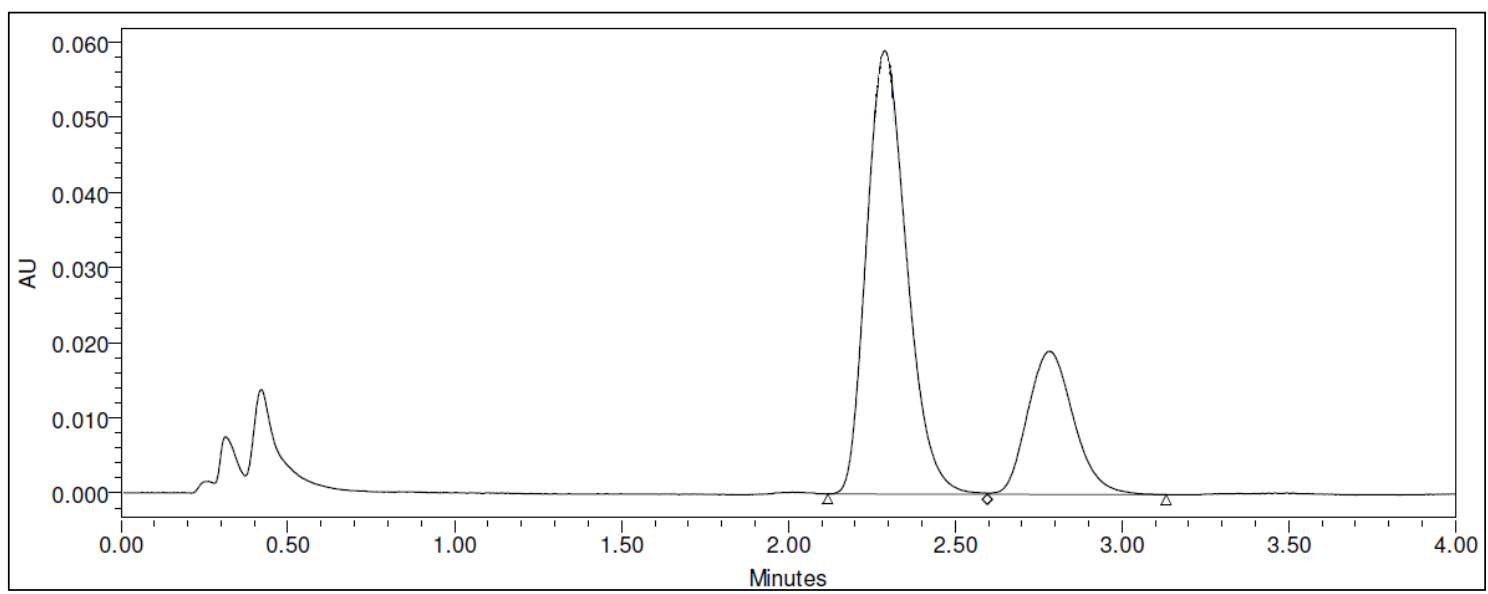

Figura 4. Exemplo de um cromatograma de solução padrão contendo $20 \mathrm{mg} \cdot \mathrm{L}^{-1}$ de permetrina com detecção em $210 \mathrm{~nm}$

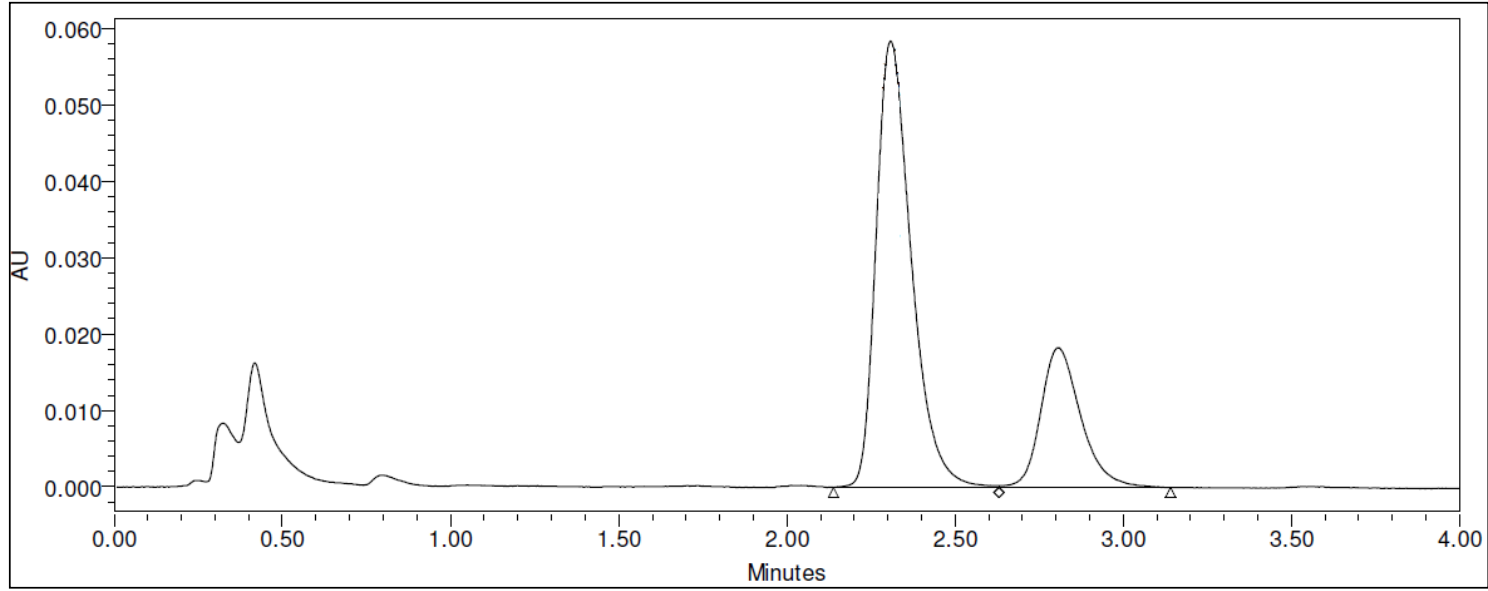

Figura 5. Exemplo de um cromatograma do extrato de permetrina obtido do tecido com deteç̧ão em $210 \mathrm{~nm}$

O processo de esgotamento industrial foi adaptado à escala laboratorial. Para maior qualidade dos resultados e garantia da repetitividade, realizou-se 0 mesmo procedimento em um total de cinco impregnações de tecidos por esgotamento. Todas as etapas do ciclo (impregnação, extração e quantificação) foram realizadas em um único dia, e o trabalho com as cinco amostras concluídas em cinco dias.

A Tabela 2 apresenta os resultados referentes às concentrações de permetrina encontradas em tecidos impregnados por esgotamento. Nessa Tabela encontram-se as médias das concentrações de permetrina para cada procedimento. 
Tabela 2. Concentrações obtidas na determinação de permetrina nos tecidos impregnados por esgotamento

\begin{tabular}{cc}
\hline Esgotamento & Média das concentrações $\left(\mathrm{mg} \cdot \mathrm{m}^{-2}\right)$ \\
\hline I & 1096 \\
II & 1011 \\
III & 913,5 \\
IV & 1119 \\
V & 1096 \\
\hline Média Total $(\mathrm{n}=5)$ & $1047 \pm 85$ \\
\hline
\end{tabular}

Devido ao seu maquinário característico, o processo de foulardagem não pode ser reproduzido em escala laboratorial. No entanto, amostras de tecidos submetidos a esse processo em uma fábrica de roupas foram cedidas para que esse estudo pudesse ser realizado.

Assim como no processo de esgotamento, os resultados foram feitos em replicatas. Nesse caso, como havia a dependência de doações de tecidos de uma fábrica colaboradora, a extração foi feita em um uma quantidade inferior de tecidos impregnados $(n=3)$. Assim como no estudo do esgotamento, todas as etapas (impregnação, extração e quantificação) foram feitas em um mesmo dia.

$\mathrm{Na}$ Tabela 3 estão apresentados os resultados referentes à quantificação da permetrina adsorvida no tecido pelo processo de Foulardagem.

Tabela 3. Concentrações obtidas na determinação de permetrina nos tecidos impregnados por foulardagem

\begin{tabular}{cc}
\hline Foulardagem & Média das concentrações $\left(\mathrm{mg}^{\mathrm{m}} \mathrm{m}^{-2}\right)$ \\
\hline I & 605,1 \\
II & 577,5 \\
III & 569,8 \\
\hline Média Total $(\mathrm{n}=3)$ & $584,1 \pm 18,6$ \\
\hline
\end{tabular}

Em uma avaliação inicial comparando os resultados obtidos pelos dois métodos de impregnação (Tabelas 2 e 3 ), pode-se observar que uma maior quantidade de permetrina (cerca de 45\%) foi adsorvida sobre a superfície do tecido no processo de esgotamento. Entretanto, para confirmação desta observação, os valores foram comparados estatisticamente, utilizando-se o teste t-Student com $95 \%$ de confiança. Em tal teste o valor de $t$ calculado foi igual a 9,025, enquanto que $o$ valor de $t$ crítico, para 6 graus de liberdade (perde-se um grau de liberdade para cada média), é igual a 1,940 (teste unicaudal). ${ }^{23}$ Deste modo, a hipótese nula foi rejeitada e considerou-se então que as quantidades de permetrina adsorvidas sobre os tecidos foram, de fato, diferentes.
Como a composição química do tecido utilizado nos dois processos foi a mesma (100\% algodão), como a concentração de permetrina utilizada no banho foi a mesma (18 g. L $\left.^{-1}\right)$ e como o procedimento de extração e quantificação para os dois tecidos foi exatamente o mesmo, pode-se afirmar que $o$ processo de esgotamento se mostrou mais eficiente para depositar permetrina sobre a superfície desse tecido do que o processo de foulardagem.

O processo de impregnação consiste em duas etapas. Uma etapa chamada de etapa "cinética" na qual, a permetrina precisa de um tempo definido para se difundir pelas fibras do tecido, impulsionada tanto pela convecção do sistema (agitação mecânica) quanto pela difusão (lei de Fick). Na outra 
etapa, denominada de "etapa termodinâmica", apenas a afinidade química entre as fibras e a espécie que esta sendo impregnada é que são consideradas (o sistema atinge 0 equilíbrio). ${ }^{24}$ Nos dois processos, certamente, a afinidade entre as fibras e a permetrina é a mesma. Entretanto, o tempo de exposição dos tecidos á solução contendo a permetrina é modificado. Deste modo, pode-se considerar que a maior eficiência na impregnação do tecido utilizando-se o processo de esgotamento está provavelmente associada ao maior tempo de exposição do mesmo à solução contendo a permetrina.

O processo de foulardagem faz uso de cilindros para comprimir mecanicamente o tecido dentro de um banho específico à altas temperaturas. Isso tende a aumentar a transferência de massa. No entanto, uma das grandes vantagens desse processo para a indústria é que ele é feito de maneira contínua. Já o processo de esgotamento, é descontínuo, e o tecido é deixado por um tempo dentro de um banho sobre agitação.

Embora a transferência de massa seja mais eficiente em um processo de foulardagem, no processo de esgotamento há um maior tempo de contato entre o tecido e o banho. ${ }^{21}$ Pode-se atribuir a esse fator, à maior eficiência na impregnação da permetrina em tecidos submetidos ao processo de esgotamento.

\section{Conclusão}

As técnicas de fixação utilizadas para a impregnação da permetrina em tecidos esgotamento e foulardagem - permitiram a adsorção de quantidades apreciáveis do inseticida sobre tecidos com composição $100 \%$ algodão. As quantidades adsorvidas foram determinadas empregando-se um método cromatográfico com limites de detecção e quantificação adequados. Assim, foi possível fazer uma avaliação entre o desempenho dos dois processos e constatou- se que, para o caso em questão, o processo de esgotamento se mostrou mais eficiente que o processo de foulardagem, uma vez que a concentração da permetrina impregnada nos tecidos submetidos a esse processo foi maior.

O estudo apresentado mostrou-se como uma valiosa ferramenta para avaliação do processo produtivo de tecidos impregnados com permetrina, por fornecer a possibilidade de reduzir custos operacionais, levando a processos mais eficientes e com menor custo.

\section{Agradecimento}

Os autores gostariam de agradecer à FAPERJ e ao CNPq pelo apoio financeiro.

\section{Referências Bibliográficas}

${ }^{1}$ Fradin, M. S.; Day, J. F. Comparative efficacy of insect repellents against mosquito bites. The New England Journal of Medicine 2002, 347, 13. [CrossRef] [PubMed]

2 Consoli, R. A. G. B.; Oliveira, R. L. Principais mosquitos de importância sanitária no Brasil, Editora Fiocruz : Rio de Janeiro, 1994 [Link]

${ }^{3}$ Côrtes, J. A. Epidemiologia - Conceitos $e$ Princípios Fundamentais. Livraria Varela: São Paulo, 1993.

${ }^{4}$ Mulla, S.; Tianyun, S. Activity and biology effects of neem products against arthropods of medical and veterinary importance. Journal of the American Mosquito Control Association 1999, 15, 133. [PubMed]

${ }^{5}$ Barreto, C. F. Aedes aegypti - Resistência aos Inseticidas Químicos e as Novas Alternativas de Controle. Revista Eletrônica Faculdade Montes Belos 2005, 1, 62. [Link] ${ }^{6}$ Carvalho, M. S. L.; Caldas, E. D.; Degallier, N.; Vilarinhos, P. T. R.; Souza, L. C. K. R.; Yoshizawa, M. A. C.; Knox, M. B.; Oliveira, C. Suscetibilidade de larvas de Aedes aegypti ao inseticida temephos no Distrito Federal. Revista de Saúde Pública 2004, 38, 623. [CrossRef] 
${ }^{7}$ Almeida, J. E. M.; Batista Filho, A. Banco de microrganismos

entomopatogênicos. Biotecnologia Ciência \& Desenvolvimento 2001, 20, 30. [Link]

${ }^{8}$ Lee, S. H., Yoon, K. S., Williamson, M. S., Goodson, S. J., Lee, M. T., Edman, J. D., Devonshire, A. L., Clark, J. M. Molecular analysis of $k d r$-like resistance in permethrinresistant strains of head lice, Pediculus capitis. Pesticide Biochemistry and Physiology 2000, 66, 130. [CrossRef]

${ }^{9}$ United States Environmental Protection Agency (U.S.EPA). Disponível em: < http://www.epa.gov/opp00001/factsheets/c hemicals/deet.htm>. Acesso em: 20 janeiro 2014.

${ }^{10}$ World Health Organization (WHO). Safety of Pyrethroids for Public Use: Communicable Disease Control. Prevention and Eradication 2005. [Link]

${ }^{11}$ Appel, K. E.; Gundert-Remy, U.; Fischer, H.; Faulde, M.; Mross, K. G.; Letzel, S.; Rossbach, B. Risk assessment of Bundeswehr (German Federal Armed Forces) permethrinimpregnated battle dress uniforms (BDU). International Journal of Hygiene and Environmental Health 2008, 211, 88. [CrossRef] [PubMed]

${ }^{12}$ Brasil. Ministério da Saúde. Manual de vigilância da saúde de populações expostas a agrotóxicos. Disponível em:<http://bvsms.saude.gov.br/bvs/publicac oes/livro2.pdf>. Acesso em: 5 abril 2014.

${ }^{13}$ Mundo Animal. Atualização Científica. Piretróides. Volume IV. Disponível em $<$ http://mundoanimal.vet.br/infomativos/pdf s/ESTUDO04.pdf>. Acesso em: 15 maio 2014.

${ }^{14}$ Soderlund, D. M.; Clark, J. M.; Sheets, L. P.; Mullin, L. S.; Piccirillo, V. J.; Sargent, D.; Stevens, J. T.; Weiner, M. L. Mechanisms of pyrethroid neurotoxicity: implications for cumulative risk assessment. Toxicology 2002, 171, 3. [CrossRef]

${ }^{15}$ Elliott, M.; Farnham, A. W.; Jones, N. F.; Needham, P. H.; Pulman, D. A.; Stevenson, J. H. Nature 1973, 246, 169. [CrossRef] [PubMed]
${ }^{16}$ Fradin, M. S. Mosquitoes and mosquito repellents: a clinician's guide. Annals of Internal Medicine 1998, 128, 931. [CrossRef] [PubMed]

${ }^{17}$ Miadoková, E.; Vlcková, V.; Duhová, V.; Trebatická, M.; Garajová, L.; Grolmus, J.; Podstavková, S.; Vlcek, D. Effects of supercypermethrin, a synthetic developmental pyrethroid, on four biological test systems. Mutation Research 1992, 280, 161. [CrossRef]

${ }^{18}$ Faulde, M. K., Uedelhoven, W. M., Robbins, R. G. Contact toxicity and residual activity of different permethrin-based fabric impregnation methods for Aedes aegypti (Diptera: Culicidae), Ixodes ricinus (Acari: Ixodidae), and Lepisma saccharina (Thysanura: Lepismatidae). Journal of Medical Entomology 2003, 40, 935. [CrossRef] [PubMed]

${ }^{19}$ Faulde, M. K., Uedelhoven, W. M., Malerius, M., Robbins, R. G. Factory-based permethrin impregnation of uniforms: residual activity against Aedes aegypti and Ixodes ricinus in battle dress uniforms worn under field conditions, and crosscontamination during the laundering and storage process. Military Medicine 2006, 171, 472. [PubMed]

${ }^{20}$ National Research Council (NRC). Health Effects of Permethrin-Impregnated Army Battle-Dress Uniforms. The National Academy Press, Washington DC, 1994.

${ }^{21}$ Associação Brasileira de Químicos e Coloristas Têxteis (ABQCT). Disponível em: <http://www.abqct.com.br/artigost/tecnolog ia textil Basica.pdf>. Acesso em: 27 janeiro 2014.

${ }^{22}$ García, E.; García, A.; Barbas, C. Validated HPLC method for quantifying permethrin in pharmaceutical formulations. Journal of Pharmaceutical and Biomedical Analysis 2001, 24, 999. [CrossRef]

${ }^{23}$ Miller, J. C.; Miller, J. N. Statistics for Analytical Chemistry, 3rd ed., Ellis Horwood PTR Prentice-Hall: Chichester, 1993.

${ }^{24}$ Twardokus, R. G.; Dissertação de Mestrado, Universidade Federal de Santa Catarina, 2004. [Link] 